\title{
Maximum likelihood estimation of the position of a radiating source in a waveguide
}

\author{
Melvin J. Hinich \\ Virginia Polytechnic Institute and State University, Blacksburg, Virginia 24061 \\ (Received 21 February 1979; accepted for publication 28 April 1979)
}

\begin{abstract}
An array of sensors is receiving radiation from a source of interest. The source and the array are in a oneor two-dimensional waveguide. The maximum-likelihood estimators of the coordinates of the source are analysed under the assumptions that the noise field is Gaussian. The Cramer-Rao lower bound is of the order of the number of modes which define the source excitation function. The results show that the accuracy of the maximum likelihood estimator of source depth using a vertical array in a infinite horizontal waveguide (such as the ocean) is limited by the number of modes detected by the array, regardless of the array size.
\end{abstract}

PACS numbers: 43.60.Cg, 43.20.Ks, 43.20.Bi

\section{INTRODUCTION}

Arrays of interconnected receivers are used to improve the signal-to-noise ratio of coherent radiation from a source located in the same waveguide as the array. In most examples, the phases of the received signals are adjusted by digital or analog methods in order to concentrate the received energy in a narrow beam with respect to the coordinate system of the array. This procedure (called beamforming) is used to estimate the direction of a point source which is radiating coherent energy at a single frequency or in a band of frequencies. Beamforming is widely used for processing arrays in radio astronomy, underwater acoustics, phased array radars, seismology, and atmospheric physics. The statistical properties of beamforming has been studied by Levin, ${ }^{1}$ Clay, Hinich, and Shaman, ${ }^{2}$ and Green, Kelley, and Levin. ${ }^{3}$

Beamforming is an essential element of the maximum likelihood estimator of source direction if the received radiation is a plane or cylindrical wave. Beamforming will result in a biased or very imprecise estimate of direction if the received wave fronts are significantly effected by reflections from the boundaries of the waveguide, or are convoluted by refraction and dispersion in the medium of propagation (see $\mathrm{Clay}^{4}$ ). Such is the case when the problem is estimating the depth of a source in an infinite stratified horizontal waveguide. Clay $^{5}$ reviews signal processing theory for such a waveguide using both horizontal and vertical arrays, and relates the statistical models to physical oceanography. The maximum likelihood estimator of source direction using a horizontal array is given by Capon et al., ${ }^{6}$ and Hinich and Shaman. ${ }^{7}$ The maximum likelihood estimator of source depth using a vertical array is given by Hinich.8, 9

This paper considers the problem of estimating the coordinates of a source in a one-or two-dimensional waveguide when the source excites a finite number of eigenfunctions of the wave operator. The boundary conditions cause a fundamental limitation of the precision the estimator irrespective of the number of receivers used in the array. This limitation is due to the limited dimensionality of the model which is induced by the physics of the system. For example, the results establish that a vertical array in an infinite horizontal waveguide (such as the ocean) need only have $M$ sensors in order to estimate the depth of a source if only $M$ modes are received. In contrast to the noise model I used in Ref. 8, this paper develops the noise field as a function of the waveguide parameters.

\section{NORMAL MODE SOLUTION OF THE WAVE EQUATION}

The steady-state solution of wave equation for an infinite horizontal waveguide is a special case of the general solution of the inhomogeneous Sturm-Louiville partial differential operator on the space $\mathcal{L}_{2}(S)$ of square integrable functions on $x \in S$, a smoothly closed and bounded subset of Euclidean space (Chap. 5, Vladimirovio). For expository purposes we first limit attention to one space dimension, and set $S=[0,1]$. For $x$ in the unit interval $[0,1]$, let $f(x)$ denote the twice continuously differentiable solution to the inhomogeneous SturmLiouville problem $L f=s\left(x-x_{0}\right)$, where the operator is defined by

$$
L f=-\frac{\partial}{\partial x} p \frac{\partial}{\partial x} f+q f,
$$

$s\left(x-x_{0}\right)$ is the intensity of a source at position $x_{0} \varepsilon S, p$ is a given continuously differentiable positive function of $x \in S, q$ is non-negative and continuous in $[0,1]$, and $f$ satisfies the following boundary conditions given $c_{i}$ $\geqslant 0, i=1, \ldots, 4$ :

$$
c_{1} f(0)-c_{2} \frac{\partial}{\partial x} f(0)=0, \quad c_{3} f(1)+c_{4} \frac{\partial}{\partial x} f(1)=0 .
$$

For steady-state processes $f(x)$ is the solution to a general wave equation (or a diffusion equation) with the given boundary conditions with normalized space units. The time component in the solution is separable from the space component.

The Sturm-Liouville operator $L$ is self-adjoint, its eigenvalues $\gamma_{1} \leqslant \gamma_{2} \leqslant \ldots$ are discrete and non-negative, and its eigenfunctions $\phi_{1}, \phi_{2}, \ldots$ are complete in $\mathcal{L}_{2}(S)$. As an example, consider the simplest model of the ocean as an acoustic waveguide, the homogeneous compressible fluid waveguide with a rigid bottom and a free 
surface. In the absence of gravity effects, the eigenfunctions for this waveguide are $\phi_{m}=\sqrt{2} \cos \gamma_{m} x$, and the eigenvalues are $\gamma_{m}=(m+1 / 2) \pi, m=1,2, \ldots$, where the units have been normalized so that the depth of the guide is one unit.

Returning to the general one-dimensional waveguide, assume that $\gamma_{1}>0$ ( $L$ is nonsingular). Then

$$
f(x)=\sum_{m=1}^{\infty}\left(s, \phi_{m}\right) \gamma_{m}^{-1} \phi_{m}(x),
$$

where

$$
\left(s, \phi_{m}\right)=\int_{0}^{1} s(x) \phi_{m}(x) d x
$$

is the inner product between $s$ and $\phi_{m}$. Moreover the eigenfunctions are orthonormal, i.e., $\left(\phi_{m}, \phi_{m}\right)=1$ and $\left(\phi_{m}, \phi_{m^{\prime}}\right)=0$ for $m \neq m^{\prime}$. When $L$ is the wave operator, the $\phi_{m}$ are called the normal modes.

Suppose that the source excites only the first $M$ eigenfunctions, i.e., the source function is a linear combination of the modes $\phi_{1}, \ldots, \phi_{M}$. The source function then can be written

$$
s\left(x-x_{0}\right)=\sum_{m=1}^{M} \theta_{m}\left(x_{0}\right) \phi_{m}(x),
$$

where $\theta_{1}, \ldots, \theta_{M}$ are unknown weights which are twice continuously differentiable functions of the source position $x_{0}$. For any $s \in \mathcal{L}_{2}(S)$, the function can be approximated to any preassigned tolerance by choosing $M$ sufficiently large, but the high modes may be so attenutated at the array that they provide negligible signal information. By the orthogonality of the eigenfunctions, it follows from (3) and (4) that

$$
f\left(x \mid x_{0}\right)=\sum_{m=1}^{N} \theta_{m}\left(x_{0}\right) \gamma_{m}^{-1} \phi_{m}(x) \text {. }
$$

Thus the received signal also lies on the $\phi_{1}, \ldots, \phi_{M}$ linear manifold.

Expressions (4) and (5) alsohold for a two-dimensional waveguide such as an ocean canyon or a fiord, and a source which excites only the first $M$ normal modes. The eigenfunctions $\phi_{m}$ satisfy the boundary conditions

$$
\alpha(\mathbf{x}) f(\mathbf{x})+\beta(\mathbf{x}) \frac{\partial f}{\partial \mathbf{n}}=0,
$$

where $\mathbf{n}$ is the external normal vector to the (piecewise smooth) boundary of the waveguide and $\alpha, \beta \geqslant 0$ are continuous on the boundary with $\alpha+\beta>0$. Moreover the operator is

$$
L=-\operatorname{div}(p \nabla)+q,
$$

where $\nabla$ is the gradient operator and div is the divergence. Estimating the source position $\mathrm{x}_{0}$ involves a linear statistical model whose structure is determined by the physics of the waveguide. The eigenfunctions of the operator $L$ constitute a "natural" basis for the statistical model.

\section{A STATISTICAL MODEL}

Assume that the source and the receiving array are in a closed one-or two-dimensional waveguide as modeled by expressions (6) and (7). The finiteness of the waveguide shapes the structure of the noise field.

Let $x_{1}, \ldots, x_{n}$ denote the positions of the receivers in the array, and as before let $x_{0} \in S$ denote the position of the source of interest. For an infinite horizontal waveguide, $x_{0}$ is the depth of the source (see Ref. 9). The coordinates $\left(x_{01}, x_{02}\right)$ of $\mathbf{x}_{0}$ are the unknown parameters which are to be estimated from a sample of the signals received by the array. If the guide is a long fiord with parallel walls, $x_{01}$ is the source depth and $x_{02}$ is the distance of the source from one wall.

Suppose that the signal has phase coherent energy in a narrow band about frequency $f_{0}$. Let $y\left(x_{i}\right)$ denote the output from the $i$ th receiver in the array which has been filtered in a narrow band about $f_{0}$. If the signal has energy in many frequency bands, the results in this paper apply to each narrowband component of the received signal.

By the linearity of the wave operator,

$$
y\left(\mathbf{x}_{i}\right)=f\left(\mathbf{x}_{i} \mid \mathbf{x}_{0}\right)+\epsilon\left(\mathbf{x}_{i}\right),
$$

where $\epsilon\left(x_{i}\right)$ is the filtered signal received at $x_{i}$ from the ensemble of noise sources in $S$. Assuming that the phases of the noise field in the narrow band around $f_{0}$ are incoherent, the signal-to-noise ratio is increased by filtering the received signal in the $f_{0}$ band.

Assume that each noise source excites at most $N$ modes, where $N \gg M$. It then follows from (4) and (5) that $\epsilon\left(\mathbf{x}_{i}\right)$ is a linear combination of the modes $\phi_{1}, \ldots, \phi_{N}$. Let $\epsilon_{m}$ replace $\theta_{m}$ in the linear combination relating $\epsilon(\mathrm{x})$ to the $\phi_{m}(\mathrm{x})$.

In order to simplify the stochastic structure of the noise field $\epsilon(x)$, assume that

$$
\epsilon(\mathrm{x})=\sum_{m=1}^{N} \epsilon_{m} \gamma_{m}^{-1} \phi_{m}(\mathrm{x}),
$$

where $\epsilon_{1}, \ldots, \epsilon_{N}$ are uncorrelated Gaussian random variables with zero mean and variances $E \epsilon_{m}^{2}=\omega_{m}^{2}$. Thus the variance-covariance matrix of the noise observed at $n$ locations $\mathrm{x}_{1}, \ldots, \mathrm{x}_{n}$ is the $n \times n$ matrix

$$
\Sigma_{x}=\Phi \Gamma_{N}^{-1} \Omega_{N} \Gamma_{N}^{-1} \Phi^{T},
$$

where $\Phi$ is the $n \times N$ matrix whose $(i, m$ th) element is $\phi_{m}\left(\mathbf{x}_{i}\right), \Gamma_{N}$ is the $N \times N$ diagonal matrix with elements $\gamma_{m}$, and $\Omega_{N}$ is the $N \times N$ diagonal matrix with elements $\omega_{m}^{2}(m=1, \ldots, N)$.

The matrix $\Sigma_{x}$ is positive semidefinite with rank at most $N$ if $n \geqslant N$. As can be seen from expression (8), there are only $N$ independent realizations from the stochastic noise process during the sampling period regardless of the number of sensors in the array. I will now show that the actual degrees-of-freedom with respect to the source parameter is $M$.

Setting the number of sensors $n=N$, suppose that the sensor locations are chosen so that $\Phi$ is nonsingular. Thus,

$$
\Sigma_{x}^{-1}=\left(\Phi^{-1}\right)^{T} \Gamma \Omega_{N}^{-1} \Gamma \Phi^{-1} .
$$

The Fisher information is the positive definite $2 \times 2$ 
matrix

$$
\mathrm{I}_{x}\left(\mathrm{X}_{0}\right)=\left(\nabla \mathrm{f}_{x}\right)^{T} \Sigma_{x}^{-1}\left(\nabla \mathrm{f}_{x}\right),
$$

where $\nabla \mathrm{f}_{x}$ is the $N \times 2$ matrix whose $i$ th row in the gradient

$$
\left(\frac{\partial}{\partial x_{01}} f\left(\mathbf{x}_{i} \mid \mathbf{x}_{0}\right), \frac{\partial}{\partial x_{02}} f\left(\mathbf{x}_{i} \mid \mathbf{x}_{0}\right)\right)
$$

at $\mathrm{x}_{0}$. Since

$$
f\left(\mathbf{x} \mid \mathbf{x}_{0}\right)=\sum_{m=1}^{N} \theta_{m}\left(x_{0}\right) \gamma_{m}^{-1} \phi_{m}(x),
$$

then from (10) and (11) we have for all $N \geqslant M$,

$$
\mathbf{I}_{x}\left(\mathrm{x}_{0}\right)=\left[\nabla \theta\left(\mathrm{x}_{0}\right)\right]^{T} \Omega_{M}^{-1}\left[\nabla \theta\left(\mathrm{x}_{0}\right)\right],
$$

where $\Omega_{M}$ is the diagonal matrix with elements $\omega_{1}^{2}, \ldots, \omega_{M}^{2}$ and $\nabla \theta\left(x_{0}\right)$ is the $M \times 2$ matrix whose $m, j$ th element is $\left(\partial / \partial x_{o j}\right) \theta_{m}\left(x_{0}\right)$.

It is clear from (12) that the elements of $I_{x}$ are independent of the array size as long as $n=N \geqslant M$. This means that at most $M$ sensors are needed for the maximum likelihood estimation of $x_{0}$, provided that the $x_{i}$ 's are chosen to make the rows of $\Phi$ linearly independent vectors. This result still holds when the variancecovariance matrix of the $\epsilon_{m}$ is not diagonal, i.e., the $\epsilon_{m}$ are correlated. In this case the $\Omega_{N}$ matrix in (12) is the $M \times M$ variance-covariance of $\left(\epsilon_{1}, \ldots, \epsilon_{M}\right)$.

For each $j=1,2$ and $m=1, \ldots, M$, let $B_{m j}$ denote the maximum of $\left|\left(\partial / \partial x_{0 j}\right) \theta_{m}\left(\mathrm{x}_{0}\right)\right|$ for $\mathrm{x}_{0} \varepsilon S$. Given a vector $a=\left(a_{1}, a_{2}\right)^{T}$ of unit length, it follows from (11) by the Schwarz inequality that

$$
\begin{aligned}
\mathrm{a}^{T} I_{x}\left(\mathrm{x}_{0}\right) \mathrm{a} & \leqslant \sum_{m=1}^{M} \omega_{m}^{-2}\left(B_{m 1}^{2}+B_{m 2}^{2}\right) \\
& \leqslant 2 \lambda^{-1} \overline{B^{2}} M,
\end{aligned}
$$

where

$$
\overline{B^{2}}=\max _{m, j}\left\{B_{m j}^{2}\right\} \text { and } \lambda=\min _{m}\left\{\omega_{m}^{2}\right\}
$$

Thus the variance of an unbiased estimator of $x_{a j}$ is bounded below by $\lambda\left(2 \overline{B^{2}} M\right)^{-1}$. For a nondiagonal $\Omega_{M}$, $\lambda$ is replaced by the minimum eigenvalue of $\Omega_{\mu}$. Thus the accuracy of the estimator of source depth using a vertical array in a horizontal waveguide is limited by the number of modes which the array detects.

\section{MAXIMUM LIKELIHOOD ESTIMATION OF $x_{0}$}

Assume that the $M$ sensor array is constructed so that the $M \times M$ matrix $\Phi=\left(\phi_{m}\left(\mathrm{x}_{i}\right)\right)$ is nonsingular. Then the model can be written

$$
y=\Phi \Gamma^{-1}\left(\theta\left(x_{0}\right)+\epsilon\right),
$$

where $\mathrm{y}=\left(y\left(\mathrm{x}_{1}\right), \ldots, y\left(\mathrm{x}_{M}\right)\right)^{T}, \theta\left(\mathrm{x}_{0}\right)=\left(\theta_{1}\left(\mathrm{x}_{0}\right), \ldots, \theta_{M}\left(\mathrm{x}_{0}\right)\right)^{T}$, and $\epsilon=\left(\epsilon\left(x_{1}\right), \ldots, \epsilon\left(x_{M}\right)\right)^{T}$. Since the $\epsilon\left(x_{i}\right)$ are normally distributed with variance-covariance matrix $\Omega_{N}$, then the first-order conditions for the maximum likelihood estimator of $x_{0}$ are

$$
\left(\Gamma \Phi^{-1} \mathrm{y}-\theta\left(\mathrm{x}_{0}\right)\right)^{T} \Omega_{M}^{-1}\left[\nabla \theta\left(\mathrm{x}_{0}\right)\right]=0,
$$

where $\nabla \theta\left(\mathrm{x}_{0}\right)$ is the $M \times 2$ matrix $\left[\left(\theta / \partial x_{a_{j}}\right) \theta_{m}\left(\mathrm{x}_{0}\right)\right]$. The maximum likelihood estimator $\hat{\mathbf{x}}_{0}$ is one of the solutions to Eq. (15) (see Chap. 5, Rao. ${ }^{11}$ )

It has been assumed that $\theta\left(\mathbf{x}_{0}\right)$ is twice continuously differentiable. In addition, assume that $\mid\left(\partial^{3}\right)$ $\left.\partial x_{0 i} \partial x_{0 j} \partial x_{0 k}\right) \theta\left(x_{0}\right) \mid$ has a finite upper bound for all $x_{0} \varepsilon S$ and $i, j, k=1$ or 2 . Given the normality of the errors in the model, it follows that as $M \rightarrow \infty, M^{1 / 2}\left(\hat{\mathbf{x}}_{0}-\mathrm{x}_{0}\right)$ is asymptotically normally distributed $N\left(0, \mathrm{I}_{x}^{-1}\left(\mathrm{x}_{0}\right)\right)$. Remember that $M$ is determined by the physical properties of the source and the medium.

\section{NONSTATIONARITY OF THE WAVEGUIDE}

It is often the case that the medium or the boundaries of the waveguide are slightly nonstationary during the period when the received signals are being filtered. In order to model the effect of this nonstationarity, assume that the filtered signal received at $x_{i}$ from a source at $x_{0}$ is given by the expression

$$
f\left(\mathbf{x}_{i} \mid \mathbf{x}_{0}\right)=\sum_{m=1}^{N} \theta_{m}\left(\mathbf{x}_{0}\right)\left[\gamma_{m}^{-1} \phi_{m}\left(\mathbf{x}_{i}\right)+u_{m}\left(x_{i}\right)\right],
$$

where $E u\left(\mathbf{x}_{i}\right)=0, E u_{m}^{2}\left(\mathbf{x}_{i}\right)=\sigma_{m}^{2}$, and $u\left(\mathbf{x}_{i}\right)$ is independent of $\epsilon\left(\mathrm{x}_{i}\right)$ for each $i=1, \ldots, M$. In other words, expression (5) is modified by adding a stochastic perturbation term $u\left(x_{i}\right)$ to $\gamma_{m}^{-1} \phi_{m}$ (see Chap. 6, Tolstoy and Clay ${ }^{12}$ ). The results given in the previous sections still hold as long as the $\theta_{m}\left(\mathrm{x}_{0}\right)$ have the previously assumed smoothness, but $\boldsymbol{\Omega}_{M}$ is replaced by $\boldsymbol{\Omega}_{M}+\Sigma_{u}$, where $\Sigma_{u}$ is the variance-covariance matrix of the perturbation terms. Assume that $\Sigma_{u}$ is nonsingular and is independent of the array geometry. The perturbation effects raises the asymptotic variances of the maximum likelihood estimators of the $x_{0 f}$, since

$$
\begin{gathered}
\mathrm{a}^{T}\left[\nabla \theta\left(\mathrm{x}_{0}\right)\right]^{T} \Omega_{M}^{-1}\left[\nabla \theta\left(\mathrm{x}_{0}\right)\right] \mathrm{a}<\mathrm{a}^{T}\left[\nabla \theta\left(\mathrm{x}_{0}\right)\right]^{T} \\
\times\left(\Omega_{M}+\Sigma_{u}\right)^{-1}\left[\nabla \theta\left(\mathrm{x}_{0}\right)\right] \mathrm{a},
\end{gathered}
$$

for $a \neq 0$ and $\nabla \theta \neq 0$. This result is hardly surprising. It is also clear that if $\sigma_{m}^{2}$ is very large for large $m$, the precision of the maximum likelihood estimator is limited as if $M$ was bounded.

\section{ACKNOWLEDGMENT}

This work was supported by the Office of Naval Research under contract.

${ }^{1}$ M. J. Levin, "Least-squares array processing for signals of unknown form," Radio Electron. Engr. 29, 213-222 (1965).

${ }^{2}$ C. S. Clay, M. J. Hinich, and P. Shaman, "Error analysis of velocity and direction measurements of plane waves using thick large aperture arrays," J. Acoust. Soc. Am. 53, 11611166 (1973).

${ }^{3}$ P. E. Green, E. J. Kelley, Jr., and M. J. Levin, "A comparison of seismic array processing methods," Geophys. J. R. Astr. Soc. 11, 67-84 (1966).

${ }^{4} \mathrm{C}$. S. Clay, "Waveguides, arrays, and filters," Geophysics 31, 501-505 (1966).

${ }^{5} \mathrm{C}$. S. Clay, "Use of arrays for acoustic transmission in a noisy ocean," Rev. Geophys. 4, 475-507 (1966).

${ }^{6} \mathrm{~J}$. Capon, R. J. Greenfield, and R. J. Kolker, "Multidimensional maximum likelihood processing of a large aperture seismic array," Proc. IEEE 55, 192-211 (1967).

${ }^{{ }^{M}} \mathrm{M}$. J. Hinich and P. Shaman, “Parameter estimation for an 
$r$-dimensional plane wave observed with additive independent Gaussian errors," Ann. Math. Statist. 43, 153-169 (1972).

${ }^{8}$ M. J. Hinich, "Maximum-likelihood signal processing for a vertical array," J. Acoust. Soc. Am. 54, 499-503 (1973).

${ }^{9} \mathrm{M}$. Hinich, "Array design for measuring source depth in a horizontal waveguide,"SIAM J. Appl. Math. 32, 800-805 (1977).
${ }^{10} \mathrm{~V}$. S. Vladimirov, Equations of Mathematical Physics, edited by A. Jeffrey (Dekker, New York, 1971).

${ }^{11} \mathrm{C}$. R. Rao, Linear Statistical Inference and its Applications (Wiley, New York, 1965).

${ }^{12}$ I. Tolstoy and C. S. Clay, Ocean Acoustics (McGraw-Hill, New York, 1966). 